\title{
Research on Application of Artificial Intelligence in VR Games
}

\author{
WANG XI ${ }^{\mathrm{a}, \mathrm{b} 1}$ \\ ${ }^{a}$ School of Design and Art, Xijing University, Xi'an, 710123, Shaanxi Province, China \\ ${ }^{\mathrm{b}}$ Keimyung University, Daegu, 42601, South Korea
}

\begin{abstract}
Purposed to promote the development of artificial intelligence (AI) technology in the game field, this article adopts the literature analysis method and the case study method, analyzes the problems with virtual reality (VR) games and explores several popular VR game cases in China and abroad before summarizing the artistic characteristics of VR games. Artificial intelligence technology has brought a new creative mode to the game industry, while providing more suitable game content for players. Further, artificial intelligence technology can equip VR games with better game experience by analyzing players' physiological and psychological information as well as players' game strategies. Compared with traditional games, VR games can deliver better interactivity, deeper immersion and higher fidelity. However, VR games impose higher requirements on device data and space scale; in other words, they must meet specific requirements to provide better game experience. VR games also have monotonous game types and high prices. In the future, the integration of VR games with artificial intelligence would generate more profound game-player experiences and richer game contents.
\end{abstract}

Keywords. Artificial Intelligence; VR games; Visual Interaction; Humancomputer Interaction; Game Experience Introduction

\section{Introduction}

This study explores the application of artificial intelligence technology in the field of virtual reality (VR) games and analyzes the artistic characteristics of VR games in terms of interactivity, immersion and fidelity, while researching the problems with VR games in such fields as equipment, space, type and price. With the addition of artificial intelligence, VR games can deliver better development in game creation, user experience and game content. Further, this study will provide research ideas and theoretical bases for the integration of VR games and artificial intelligence technologies. This article also refers to the following three articles: "A Survey of Visual, Mixed, and Augmented Reality Gaming"[1], "Virtual Reality in Multiplayer Carrom Game with Artificial Intelligence"[2], "Artificial Neural Network in Virtual Reality: A Survey"[3]. The above three articles are all researches on VR games or AI algorithms from the field of computer and AI technology. Both the research methods

\footnotetext{
${ }^{1}$ Corresponding Author: Wang Xi, Xijing University, Chang'an District, Xi'an City, Shaanxi Province,
} China, 710123. E-mail: 1308573796@qq.com. 
and the research steps have strong practicality, but these articles ignore the aesthetics of the game screen. It cannot study the current situation of VR games from an artistic perspective as well as it does not predict the development trend of the combination of VR games and AI technology. The above shortcomings are exactly what this article is about. this article will look at the combination of VR games and AI technology from an artistic perspective, hoping to bring new inspiration to these two fields.

The article adopts the literature analysis method and the case study method. The literature analysis method mainly collects data and materials of game companies for analysis and comparison. The case study method mainly studies the price, equipment, types of a specific game, and gives suggestions on the direction of the game development, as well as provides a theoretical reference for the game industry. This study is divided into four parts. The first part offers an overview of the current development of artificial intelligence technology, the second and third parts describes the characteristics and problems of VR games, and the last part explores the impact of artificial intelligence technology on VR games.

\section{Development of artificial intelligence}

As a branch of computer science, artificial intelligence attempts to explore the essence of intelligence and develop a new type of intelligent machines that can react in the similar way to human intelligence, covering such fields as robotics, language recognition, image recognition, nature language processing and expert systems. Over recent years, with the development of computer technology, artificial intelligence technology has developed rapidly. Meanwhile, it has spawned a huge industrial development space. Chinese government has also issued a number of policies to support, guide and standardize the artificial intelligence industry. For example, in July 2017, the State Council of China promulgated the "Development Plan for NewGeneration Artificial Intelligence", which stated the direction and industrial layout of artificial intelligence in China, so that the application can reach the global advanced level and China can become one of the important centers of global artificial intelligence. [4].

Under this background, VR games have built a novel kind of visual and tactile presentation technology system. With the help of artificial intelligence technology, traditional games can achieve virtual space and strong immersion. As a result, the excellent experience enables VR games to quickly erode some of the territory of traditional game industry, which is encountering the ever-expanding ambitions and aggressive challenges of VR games and brand-new opportunities for industrial development.

\section{Features of VR games}

Ranging from the simple rock-paper-scissors game and Prisoner's Dilemma to more complex Go games and Blizzard's popular game StarCraft, the most fascinating part of games is that they can always provide some interesting and unknown challenges, because the essence of games lies in how to figure out the next action in a complex scene, so as to achieve the final victory as defined by the games. [5] The game industry 
has made tremendous changes so far. For example, game props have evolved from buttons and keyboards to helmets and handles, and game modes have been graded from the simple two-dimensional human-machine interaction to the full-view advanced interactive mode for people currently. Now, VR games have set off another climax for the game industry. Since 2016, VR games have made a rapid growth. According to the "China Game Industry Report" released at the 2019 China Game Industry Annual Conference, "The actual sales revenue of the Chinese game market in 2019 is 230.88 billion yuan, with an increase of $7.7 \%$ year-on-year; and the number of game users in China reaches 640 million, with an increase of $2.5 \%$ year-on-year. Specifically, AR games' revenue exceeds 70 million yuan, with an increase of $64.3 \%$ year-on-year, and its user scale tops about 1.4 million, the year-on-year growth is close to $15 \%$; VR game revenue is 2.67 billion yuan. With a year-on-year growth of $49.3 \%$, VR game users has reached 8.3 million, with an increase of 22\% year-on-year." [6] Given the growth rate of VR game users is far greater than that of overall game users, VR games showcase unparalleled advantages over the traditional games.

First, VR games can deliver better fidelity and reduction, because they use various devices to create a 360-degree visible full-view space in users' minds, so as to simulate a real scene, which is different from the two-dimensional plane space generated in traditional video games. In a space with a real sense of depth, players can enjoy scenes at any angle, where players watching the plots can get a more realistic feeling. In addition, VR games can render better light changes, surrounding sounds and better restoration of the information on the objective world. [7]

Second, VR games can achieve better immersion effects. From the users' views, VR games can provide first-person perspective and multiple perspectives. With the help of sound, light, electricity and other technologies, users' physiological and psychological feelings can be integrated into a specific game environment, so that they can experience comprehensive hearing, vision, tactile and even olfactory content. By creating a fascinating and fantasy world, such an environment is almost not affected by external factors, such as light and sound. In the objective world, this technology can give users a better sense of immersion

Third, VR games can realize better interactivity. In traditional games, users make interaction through screens and sounds, which are actually simple signal feedbacks on senses, because such means ignore the interaction between games and players' body movements. [8] Therefore, the game experience is not delicate enough. However, VR games can comprehensively upgrade the interactive system by taking a more advanced method, which can perform different interactions for each change of users and provide them with different game responses in movements, such as position moves, gesture changes and visual transitions. Due to richer and more delicate interactivity, such interactions can render more real and more interesting gaming experiences. Figure 1 illustrates the interaction process between VR games and players. 


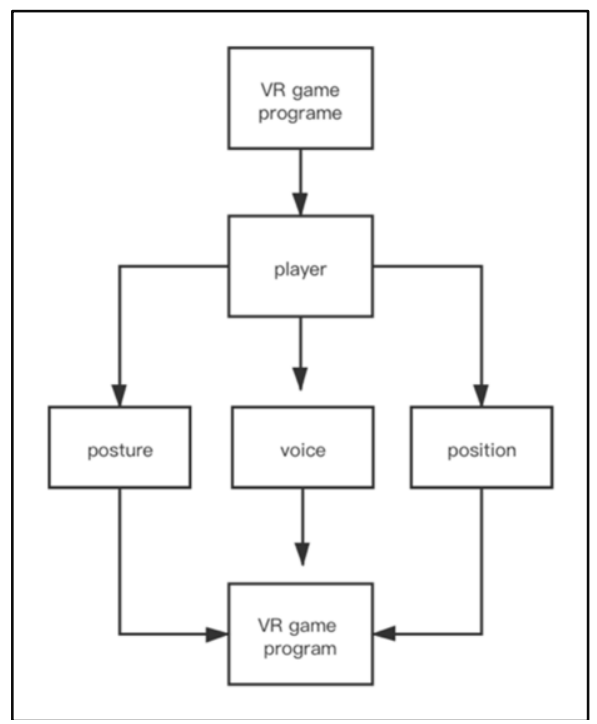

Figure 1. VR games have better interactivity

\section{Problems with VR games}

While VR games demonstrate a huge advantage in terms of experience compared to traditional games, the following limitations have to be overcome in their further development.

First, device restrictions: VR games require multiple sets of devices to complete the whole game. Especially, heavy requirements are imposed on data transmission, image resolution, somatosensory vibration, etc., thus raising the threshold for VR games' entry. Currently, mainstream VR game devices include Oculus Rift, HTC Vive and Sony PS4-VR. These high-end games also need equipment for positioning, audio, vibration, in addition to VR helmets and gamepads. Compared with the simple devices in traditional video games, the importance of VR game equipment is self-evident. However, too many devices are not easy to deal with, since the game preparation process would be too long and the tedious testing processes of game equipment would also test the patience of gamers who are always eager to dive into games. Table $\mathbf{1}$ illustrates the different types of VR equipment and their characteristics. Obviously, the complexity of the device is limiting the development of VR games.

Table 1. Equipment required for VR games.

External VR

helmet

All-in-one VR helmet
The VR display helmet relies on an external computer, host and other equipment to run on the system. The content of the platform has the highest technical content. Representative products: Oculus, HTC VIVE, etc.

The VR platform that integrates the content platform and the display device is independent and flat. And the platform strikes a balance between portability and functionality. Representative products: Little Black Spirit Mirror and others. 


$\begin{aligned} & \text { Smartphone VR } \\ & \text { glasses }\end{aligned}$
$\begin{gathered}\text { Smart glasses are used as a display device to run the system. } \\ \text { The platform has the best portability. But the content technology is } \\ \text { at a relatively low level. Representative products: Gear YR, Storm } \\ \text { Mirror, etc. }\end{gathered}$
$\begin{aligned} & \text { Shooting } \\ & \text { equipment } \\ & \text { Multiple cameras form a seamless 360-degree panoramic } \\ & \text { virtual reality camera. Representative products: GoPro, Jaunt VR } \\ & \text { NEO, etc. }\end{aligned}$
$\begin{aligned} & \text { Operating } \\ & \text { equipment }\end{aligned}$
$\begin{aligned} & \text { Such platforms include gamepads, steering wheels, and } \\ & \text { monitoring } \\ & \text { equipment }\end{aligned}$
$\begin{gathered}\text { Infrared monitoring cameras, universal treadmills, gesture } \\ \text { Others }\end{gathered}$
$\begin{gathered}\text { simpturing gloves and other devices used to monitor user behavior. } \\ \text { Source: the 2016 China Virtual Reality (VR) Industry Research Report by iResearch }\end{gathered}$

Source: the 2016 China Virtual Reality (VR) Industry Research Report by iResearch

Second, price restrictions: generally expensive, the devices of VR games affect their expansion to more users. In addition, VR games have a longer production cycle and more complicated manufacturing procedures than traditional video games. Moreover, the resulting game screens are more beautiful in VR games, thus further driving up the price. According to the Steam game website, as of June 2020, the average price of the top ten games listed on the site exceeded 120 yuan. The No.1, Beat Saber, was priced at 90 yuan. The Second half-life, Alyx, was at 163 yuan, and the fifth-ranked game, Tripwire Complete Bundle, is at 568 yuan. As a result, the excessive price weakens the interest of players to a certain extent, thus dwarfing the development of VR games.

Third, space constraints: traditional video games require game consoles or computers, monitors, keyboards, and handles or joysticks to provide players with a higher level of participation. VR games require not only VR headsets, glasses, joysticks, monitors and audio devices, but also require positioning systems, cameras and vibration systems. In order to pursue the realism and immersion for games, some game players even customize special seats, platforms and other equipment. large space is required to accommodate the equipment, a factor that often restricts the development of the VR games. Take the HTC ViveVR device as an example: officially, the game requires a space of $3.5 \mathrm{~m} \times 3.5 \mathrm{~m}$. For the setting of the scale in the room, the game area must be at least $2 \mathrm{~m} \times 1.5 \mathrm{~m}$, in order to experience a better virtual game. For example, Table 2 shows the space required by different brands of VR equipment. For young players, such an environment requirement will limit their desire to purchase VR gaming equipment.

Table 2. Space supported by different brands of VR glasses.

\begin{tabular}{ll}
\hline Brand & largest space supported \\
\hline HTC-Vive Pro & $6 \mathrm{M}^{*} 6 \mathrm{M}$ \\
Sony-Play Station & $3 \mathrm{M}^{*} 3 \mathrm{M}$ \\
Huawei-NOLO CV1 Air VR & $5 \mathrm{M}^{*} 5 \mathrm{M}$ \\
Oculus-Quest VR & $3 \mathrm{M}^{*} 3 \mathrm{M}$ \\
\hline
\end{tabular}

Fourth, the limited types of VR games. The types of VR games are not as rich as the traditional games. VR games are more widely developed for first-perspective games. 
Simulated single-player games are less involved in strategy games. Compared with wide selection of the traditional games in game stores, there are not rich types of VR games. As shown on the Steam game website, as of June 2020, although the platform had released more than 3500 VR games, the game types are slightly narrowed, because most of them are first-person ones and there are almost no strategy games available. Few game types raise obstacles to the further development of VR games.

\section{The impact of artificial intelligence on VR game}

The intervention of artificial intelligence (AI) will transform VR games. First, AI can be used to analyze users' usage habits and separately change the game progresses and rhythms, if VR games utilize artificial intelligence technology to measure game players' physical state, usage habits, etc. Moreover, AI can even test players' responses to game pressures by detecting changes in their breathing and blood pressures. Based on players' physiological and psychological data in the process of VR games, the game programs can match players' physical and psychological fitness better. For example, 18-year-old users and 30-year-old ones would show obvious physiological differences in their personal reaction speeds and stress resistances for the same game. In this case, artificial intelligence may follow the analyzed data and difficulty levels. Tensions and even changes in space and light of games may be re-allocated to maximize the satisfaction of different users. For example, in Figure 2, artificial intelligence technology modifies the game program by analyzing player data.

Figure 2. Artificial intelligence changes the course of the game:

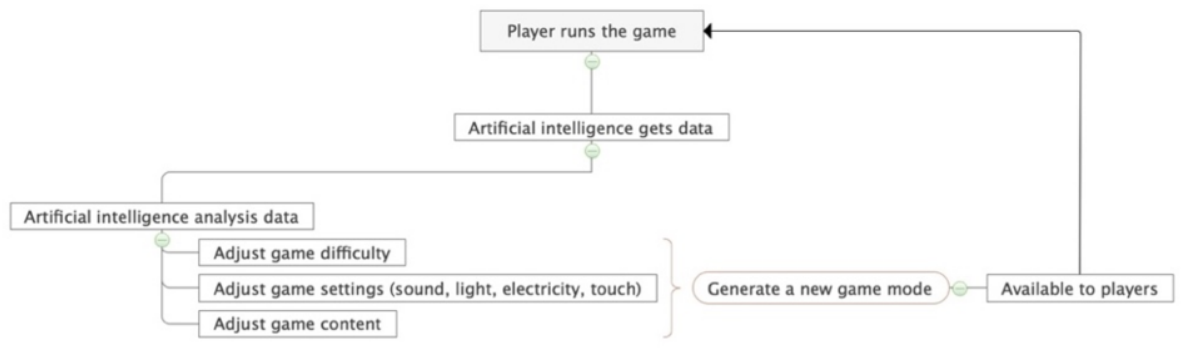

Second, artificial intelligence can automatically correct the defects in a game based on the data on game players. By actively learning the wisdom of game players, artificial intelligence may also provide a direction for the development of video games. With Alpha Go developed by Google and artificial intelligence developed by Blizzard, AI players have defeated human professional players. By studying the human thinking mode to explore a thinking process that can defeat humans, a deep simulation of the biological nervous system in the human brain is conducted. Similarly, many organizations are connected to each other through a series of associations, thereby forming a neural network-like processing mode to deal with the faced problems accurately and quickly. [9]. In short, artificial intelligence can learn human game thinking, correct its own defects and develop better game strategies. 
Third, artificial intelligence can quickly collect materials based on the content and creativity of games to help with game development. In terms of game production, artificial intelligence can actively search for relevant messages based on the key information provided by the developers as well as the required data, such as pictures, colors, light sources and sounds. Based on the key information available, artificial intelligence technology can independently complete the conversion of styles and scenes. Further, by taking advantage of artificial intelligence technology, designers can configure the characteristics of game scenes in the game development platform. Similarly, based on game development and running procedures, designers can be provided with a good window editing platform. And game test platforms can help game developers complete game development. [10]

Fourth, artificial intelligence technology can improve the performance and size of VR equipment. With the help of high-speed digital technology, VR devices will be able to get rid of heavy computing burdens, store a large amount of data in the clouds for downloading at any time, and reduce the computing tasks of devices. [11] On this basis, the volume and weight of devices can also be cut and the game content can be displayed in a more lightweight way. Then, lightweight equipment can achieve more ergonomic designs and get rid of the heavy and awkward appearance of VR equipment. If full of modern styles, VR equipment will strongly attract young people and play an important role in the development of mixed reality (MR) technology.

\section{Conclusion}

By integrating artificial intelligence and VR technology in the field of games, the development of the game industry will be accelerating, and the transformation of digital art and technology will be facilitated. With diversified game contents, the forms are becoming more abundant as well, with intelligent game modes appearing. In terms of intrinsic values, the development of artificial intelligence technology meets the requirements of players for higher levels of game art. In terms of extrinsic values, artificial intelligence technology provides a richer production method for VR games. Firstly, VR games, as a new development type in the game industry, have brought new art forms, because they provide a virtual space environment and render more real visual effects and psychological feelings. Relying on the development of artificial intelligence technology, VR games can analyze the habits of game users to adapt the game rhythms to users for more practical game changes. Secondly, the artificial intelligence system in games can acquire game-players' strategies through human-computer interaction and independent learning. Then, it may carry out the corresponding methods to fix the deficiencies in games. Thirdly, artificial intelligence can independently search for the materials needed to help designers develop games. The rapid evolvement of artificial intelligence technology and the integration of VR games with unique AI operation modes will create more attractive game content and promote the further growth of the game industry. 


\section{References}

[1] M. Z. Khan, F. Hassan, M. Usman, U. Ansari and S. Noor, "Virtual Reality in Multiplayer Carrom Game with Artificial Intelligence," 2018 12th International Conference on Mathematics, Actuarial Science, Computer Science and Statistics (MACS), Karachi, Pakistan, 2018, pp. 1-5, doi: 10.1109/MACS.2018.8628394.7.

[2] Thomas, Bruce. (2012). A survey of visual, mixed, and augmented reality gaming. Comput. Entertain. 10. 3:1-3:33. 10.1145/2381876.2381879. - Cavazza, M. Al in computer games: Survey and perspectives. Virtual Reality 5, 223-235 (2000). https://doi.org/10.1007/BF01408521.

[3] Sharma, Greeshma \& Chandra, Sushil \& Venkatraman, Saraynya \& Mittal, Alok \& Singh, Vijander. (2016). Artificial Neural Network in Virtual Reality: A Survey. International Journal of Virtual Reality. 15. 44-52. 10.20870/IJVR.2016.15.2.2873.

[4] Wisdom Bud. Global Pattern of Industrial Intelligence: Future Trends and China's Potential[J]. Economic Theory and Economic Management,2019(10):2.

[5] Alfonsina Pagano, Augusto Palombini, Guido Bozzelli, Maurizio De Nino, Ivana Cerato, Stefano Ricciardi. Arkae Vision VR Game: User Experience Research between Real and Virtual Paestum[J]. Applied Sciences,2020,10(9).

[6] Joe Chen. 2019 China Game Industry Report: VR game revenue 2.67 billion yuan. [EB/OL] VR Home. 2019-12-27 10:59.

[7] LUCAS Simon, Shen Tianyu, Wang Xiao, Zhang Jie. General artificial intelligence for games based on statistical forward planning algorithm[J]. Journal of Intelligent Science and Technology,2019,1(03):219-227.

[8] Wang Xiaohan.VR game design and implementation analysis[J]. Digital World,2020(03):55-56.

[9] Cao Kunze. Artificial intelligence and its application in the field of games[J]. Science and Technology Communication,2020,12(08):143-144.

[10] Zhao Yue. Design and implementation of 3D game development platform based on artificial intelligence characters[J]. Computer fan,2018(05):207.

[11] He Shucun. Radio and TV game service platform based on cloud computing technology[J]. Science and Technology Communication, 2015, 7(10):145-146. 